\title{
BMJ Open Pharmacokinetics and complementary evaluation system-based guidance on prophylaxis of paediatric patients with haemophilia A in China with Kovaltry: protocol of the LEAP study
}

Huang Kun (1D , ${ }^{1}$ Weiqun Xu, ${ }^{2}$ Min Zhou, ${ }^{3}$ Xiaojing Li, ${ }^{4}$ Zhongjin Xu, ${ }^{5}$ Yongjun Fang, ${ }^{6}$ Changgang Li, ${ }^{7}$ Zhenping Chen, ${ }^{8}$ Runhui $\mathrm{Wu}^{1}$

To cite: Kun H, Xu W, Zhou M, et al. Pharmacokinetics and complementary evaluation system-based guidance on prophylaxis of paediatric patients with haemophilia $A$ in China with Kovaltry: protocol of the LEAP study. BMJ Open 2021;11:e048432. doi:10.1136/ bmjopen-2020-048432

- Prepublication history and additional supplemental material for this paper are available online. To view these files, please visit the journal online (http://dx.doi.org/10.1136/ bmjopen-2020-048432).

Received 27 December 2020 Accepted 14 June 2021

Check for updates

(C) Author(s) (or their employer(s)) 2021. Re-use permitted under CC BY-NC. No commercial re-use. See rights and permissions. Published by BMJ.

For numbered affiliations see end of article.

Correspondence to Dr Runhui Wu; runhuiwu@hotmail.com and Dr Zhenping Chen; chenzhenping@outlook.com

\section{ABSTRACT}

Introduction Haemophilia $A$ is a rare inherited bleeding disease caused by the deficiency of coagulation factor VIII (FVIII). The main treatment protocol is to administer regular exogenous FVIII concentrate infusions. With the discovery of variability in individualised pharmacokinetics (PK) and bleeding phenotype, the previous weight-based approach needs to be replaced by more advanced PKtailored prophylaxis with an accurate evaluation system. In this study, we combine individualised PK profiles and a complementary evaluation system to guide prophylaxis in paediatric patients with haemophilia $A$.

Methods and analysis This is a single-centre, prospective single-arm study. The aim of this study is to assess the effectiveness of a new strategy combining PK and a complementary evaluation system to treat haemophilia A in Chinese paediatric patients. Sixty paediatric patients with haemophilia will be recruited. After PK testing, they will receive a PK-guided stepup prophylaxis in the next 2 years. The dosing regimen will be determined according to individualised PK profiles and complementary evaluation findings. Related indicators at the end of the study will be compared with the values at treatment initiation to examine the effectiveness of this new strategy. The demographic data of the investigated patients will be summarised by descriptive statistics. Quantitative data will be described by summary statistics, including arithmetic median, range, mean and arithmetic SD. Analyses will use t-test to compare indicators such as bleeding rate and imaging score at both ends of the study as well as during follow-up. Ethics and dissemination The study has been approved by the Ethics Committee of Beijing Children's Hospital (Number 2020-Z-095). The findings will be presented at international meetings such as World Federation of Hemophilia World Congress. Related manuscripts will be submitted to peer-review journals such as Blood and Hemophilia.

Trial registration number ChiCTR2000037821; Preresults.

\section{INTRODUCTION}

Haemophilia A is an X-linked inherited bleeding disorder due to the deficiency of
Strengths and limitations of this study

- This study will be the first to combine pharmacokinetic and complementary evaluation in haemophilia therapy.

- The complementary evaluation could provide better joint protection by detecting preclinical lesions.

- The small sample size may limit statistical power for further exploratory analyses.

coagulation factor VIII (FVIII). Patients with haemophilia have spontaneous bleeds in muscles or joints, which could cause joint disfunction or even death. The main treatment option for haemophilia is to administer regular exogenous FVIII infusions. Compared with on-demand therapy, prophylaxis has been considered an optimised therapy regimen to help patients live a normal life, with enhanced ability to decrease bleeds and maintain the function of joints. ${ }^{1}$ Prophylaxis is considered the standard treatment of haemophilia in paediatric patients and should be started as soon as possible once prophylaxis is proposed..$^{2}$

The first prophylaxis regimen was proposed in Sweden and is also known as "standard prophylaxis'. ${ }^{3}$ It aims to keep the trough FVIII level of patients with haemophilia A above $1 \mathrm{IU} / \mathrm{dL}$ by giving them regular exogenous FVIII concentrate injections (20-40 IU/ $\mathrm{kg}$, three times per week or every other day). The experience of the Malmo protocol in more than 50 years has revealed its clinical effectiveness in treating patients with haemophilia. ${ }^{4}$ However, the Malmo protocol has not been widely used in other regions due to the massive consumption of and insufficient access to FVIII concentrate as well as the heavy burden of frequent intravenous 
injections. Therefore, other prophylaxis protocols have been developed, including the middle dose prophylaxis in the Netherlands (15-25 IU/kg, 2-3 times per week) and the stepup prophylaxis proposed by Canada. ${ }^{56}$ In some developing counties, such as China and India, even low-dose prophylaxis was shown to greatly decrease bleeding compared with on-demand therapy. ${ }^{7}$

Currently, prophylactic regimens are determined by a standard weight-based approach, which may cause underdosage or overdosage because of the variability of both FVIII pharmacokinetic (PK) profiles and bleed phenotypes among different patients with haemophilia. According to Chen et al FVIII's half-life time varies from 5.52 to 20.02 hour, while in vivo recovery varies from 1.2 $\mathrm{IU} / \mathrm{kg}$ to $3 \mathrm{IU} / \mathrm{kg}^{8}{ }^{8}$ Other studies also confirmed great individual variability in PK profile. ${ }^{9} 10$ Thus, single weightbased prophylaxis may cause either extra bleeds and joint disfunction due to insufficient treatment or unnecessary FVIII concentrate waste with overdosage. Therefore, it has been recommended that individualised PK profiles should be employed for determining the patient's dose and frequency of routine prophylaxis. In the past, it was hard to obtain individualised PK profiles because of the heavy burden of up to 10 time points after a long washout period and single-dose infusion. ${ }^{11}$ With the application of the Bayes approach to population PK (popPK) in haemophilia, it is currently possible to use blood samples collected at only 2-3 time points to determine individualised PK profiles. ${ }^{11}$ According to Iorio $e$ al, the popPK method is a practical and accurate way to predict individualised PK profiles. ${ }^{12}$ Previous studies have revealed the advantages of PK-tailored prophylaxis in haemophilia treatment. ${ }^{13}$ In addition, online PK dosing tools such as Web Accessible Population Pharmacokinetics (WAPPS) have been recommended by official organisations to guide routine prophylaxis according to individualised PK profiles. $^{11}$

Besides variability in PK profiles, different bleed phenotypes among patients also need to be taken into consideration in routine therapy. Although Collins et al clearly demonstrated that break-through bleeds in prophylaxis are correlated to weekly time spent with low FVIII levels, some patients with high-trough FVIII levels in daily prophylaxis still suffer from bleeds, especially those with target joints. ${ }^{14}$ According to the sports guidelines for haemophilia, patients with target joints need to keep higher FVIII levels in the same sport compared with those without joint disfunction. In a Dutch study involving more than 400 patients with haemophilia, it seemed that only trough FVIII levels reached $12 \mathrm{IU} / \mathrm{dL}$ should the number of target joints decrease to zero. ${ }^{15}$ The target trough FVIII level to reach the goal of zero bleed was $15 \mathrm{IU} / \mathrm{dL}$ in another study. ${ }^{16}$ Besides the joint state, Den Uijl et al also suggested that multiple targets should be considered in determining the routine prophylaxis regimen, including physical activity, the quality of life and cost-effectiveness. ${ }^{16}$ The haemophilia care team of Beijing Children's Hospital started a study named CHIPS (Chinese Hemophilia
Individualized Prophylaxis Study) in 2016 to explore an evaluation system for paediatric patients with haemophilia, which includes multiple targets such as joint structure assessed by MRI and ultrasound (US) scores, joint function evaluated by Haemophilia Joint Health Score (HJHS) scores, the quality of life assessed by Canadian Hemophilia Outcomes-Kids Life Assessment Tool scores and other aims and scaling scores. ${ }^{17}$ In this study, some patients could keep the bleeding rate at 0 in step 1, while others still suffered from frequent bleeds even in step 4, which indicated the variability of bleed phenotype leads to a difference in target trough FVIII levels in routine prophylaxis.

Although some products involving new mechanisms to treat haemophilia are available, most patients around the world are still taking FVIII concentrate for routine prophylaxis, and this situation would not change for a long time. ${ }^{2}$ How to use PK data and a complementary evaluation system to individualise prophylaxis in patients with haemophilia, achieving better clinical outcomes and reduced cost, remains a vital question that needs to be addressed urgently.

\section{STUDY OBJECTIVES}

\section{Primary objectives}

1. To evaluate the effect of PK-based and complementary evaluation system-based instructions for prophylaxis in paediatric patients with haemophilia A (according to US and/or MRI findings).

2. To establish a popPK model for Kovaltry suitable for paediatric patients with haemophilia A in China.

\section{Secondary objectives}

1. To study the efficacy and safety of the prophylactic regimen under the guidance of PK and complementary evaluation system.

2. To evaluate the PK parameters of paediatric haemophilia A patients in China administered Kovaltry products in China.

\section{METHODS AND ANALYSIS}

\section{Ethics and dissemination}

The study was approved by the ethics committee of Beijing Children's Hospital (Number 2020-Z-095). Written informed consent was obtained from each enrolled patient and their legally authorised guardians. The SPIRIT list of this study would be available as supplementary files.

\section{Study design}

This is a multicentre, prospective single-arm study, including two stages from January 2021 to January 2024.

Stage $\mathbf{I}$ is the popPK period lasting for 6 months. The enrolled paediatric patients will be treated with Kovaltry according to current clinical situation, and the therapeutic regimen and bleeding situation will be recorded. PK indicators will be measured comprehensively. 


\section{PopPK model building and verification}

At this stage, after obtaining the individual PK information of paediatric patients, the PK and personal information of 30 paediatric patients (aged 1-18 years) will be included in WAPPS to generate the Kovaltry popPK model including the data of paediatric patients in China; meanwhile, the individual PK information of another 30 paediatric patients in China not involved in modelling will be included for external verification to ensure the accuracy and availability of the model. Considering the balanced distribution of paediatric patients in various age groups, age distribution for modelling and verification will be 1-6, 6-12 and 12-18 years old. All enrolled paediatric patients should undergo PK testing prior to the trial period. A washout period of at least 72 hours will be retained before PK testing. A single dose of $50 \mathrm{IU} / \mathrm{kg}$ coagulation FVIII concentrate (Kovaltry, BAY81-8973) will be infused, with blood samples collected at different time points before and after infusion to determine FVIII concentration. Blood samples will be taken within half an hour before infusion, and at 1 hour, 3 hours, 9 hours, 24 hours, 48 hours and 72 hours after infusion, centrifuged and tested. ${ }^{18}$ PK parameters will be obtained through the WAPPS-Hemo team. ${ }^{12}$

\section{Data collection}

After enrolment, patients' data on prophylaxis with Kovaltry in the first 6 months will be collected as baseline data in this study.

Stage II is the clinical stage lasting for 2 years. Patients will receive joint assessment and trough FVIII level test every 3 months and PK monitoring every 6 months.

All eligible patients will receive a dose-escalation prophylactic regimen guided by the results of $\mathrm{PK}$ and a complementary evaluation system, including four steps (the first step would be decided according to patients' individualised trough FVIII level in their routine prophylaxis):

Step 1: Maintained trough FVIII concentration=1-2 IU / dL.

Step 2: Maintained trough FVIII concentration=2-3 IU / dL.

Step 3: Maintained trough FVIII concentration=3-4IU / dL.

Step 4: Maintained trough FVIII concentration=4-5 IU/ dL.

Step 5: Maintained trough FVIII concentration $>5$ IU/ dL.

In the above steps, the specific dose and frequency of dosing are not stipulated. The investigators will jointly decide a therapeutic regimen with the subject based on comprehensive assessments and instructions of WAPPSHemo PK, the patient's needs for quality of life and other specific conditions.

Prophylactic administration in all eligible patients will be initially (at the seventh month) evaluated as insufficient' according to specific criteria (table 1), and the trough FVIII levels of their current prophylaxis will be upgraded to the trough concentrations at the corresponding time. This protocol aims to combine PK and a complementary evaluation system to instruct patients to receive prophylaxis and help them further control bleeding, protecting joint function and improving the quality of life.

\section{Study population}

Inclusion criteria

1. Severe haemophilia A (FVIII: C<1\%), aged 1-18 years.

Table 1 Escalation criteria determined by the complementary evaluation system

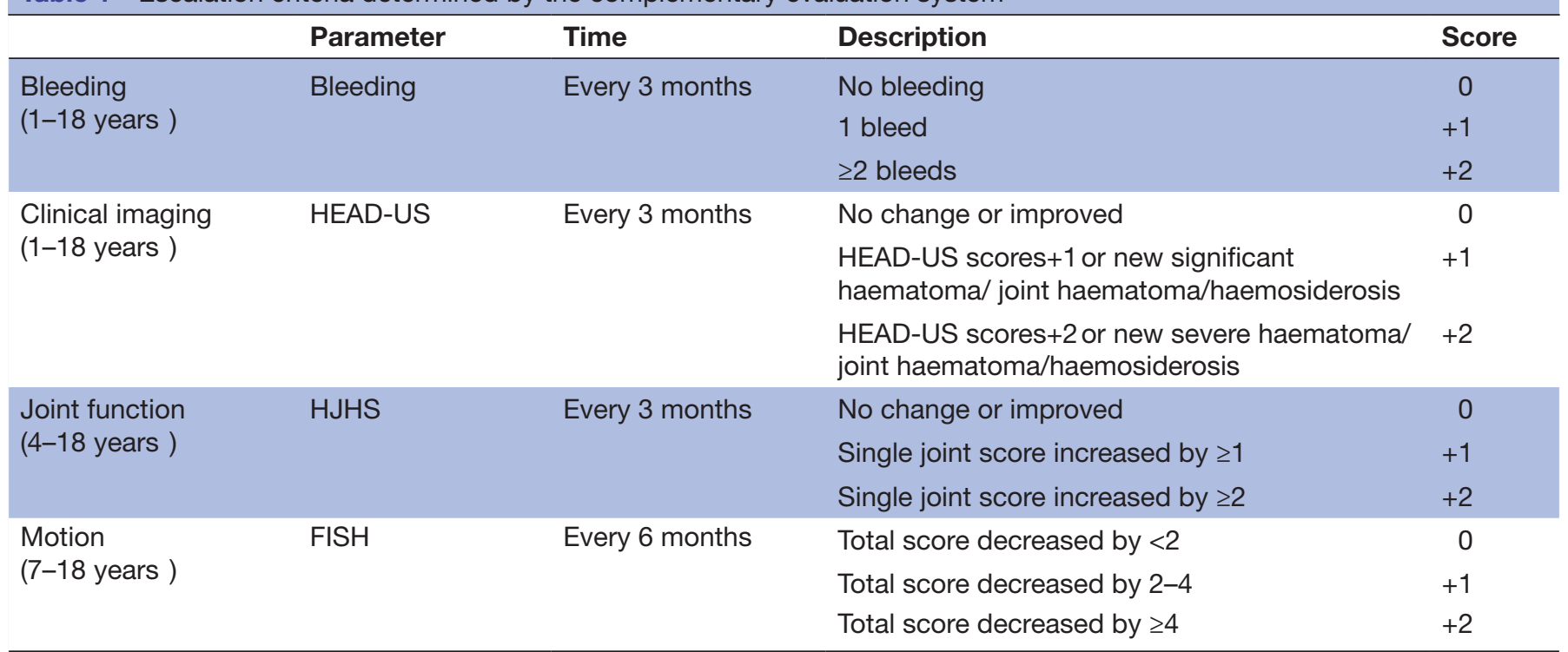

Evaluation: $<2$ points:maintain the prophylactic dose; $\geq 2$ points: increase the prophylactic dose into the next step.

FISH, functional independence score in haemophilia; HEAD-US, Hemophilia Early Arthropathy Dection with Ultrasound; HJHS, Haemophilia Joint Health Score. 
2. A history of knee, elbow or ankle bleeding.

3. $>50$ exposure days (calculated from previous treatments with FVIII products).

4. No FVIII inhibitors at enrolment.

5. Regular clinical visits and medical records available.

6 . Informed consent from the legal guardians of patients before enrolment.

7. Prophylaxis with Kovaltry being administered at the time of enrolment and baseline data on prophylaxis with Kovaltry for at least 6 months prior to phase I available.

\section{Exclusion criteria}

1. Other haemorrhagic diseases such as von Willebrand disease (VWD).

2. Generation of FVIII inhibitors: $>0.6 \mathrm{BU}$ (confirmed by two separate tests).

3. A previous history of inhibitors and presence of FVIII inhibitor at any time in the study period.

4. Planning to participate (or previous involvement) in other Kovaltry-related studies, other interventional studies or any studies expected to affect the study protocol.

5. Using other FVIII concentrates for routine prophylaxis.

\section{Sample size}

According to the guidance of the WAPPS team and the number of potential patients available in our centre, the PK data of 60 paediatric patients with haemophilia A would be sufficient for this study. In addition, due to the novelty of this study, the sample size could not be estimated through previous studies.

\section{Study endpoints and outcomes measures}

Primary endpoints

1. Percentages of MRI/US scores of joints improved/unchanged from baseline.

2. A valid Kovaltry popPK model established for patients with paediatric haemophilia A in China

\section{Second endpoints}

1. Annual bleeding rate, annual joint bleeding rate and annual target joint bleeding rate.

2. Bleeding rates will be calculated according to the routine electronic record of patients.

3. Joint function (HJHS).

4. Joint structure (X-ray Pettersson score ).

5. Motion (functional independence score in haemophilia $>7$ years of age).

6. Quality of life.

7. Consumption and therapeutic dose regimen of Kovaltry.

8. Assessment of family disease burden.

9. Treatment compliance and reasons for noncompliance of patients in various age groups.

\section{Escalation criteria}

According to the criteria detailed in table 1, the prophylactic dose would increase into the next step with a score $\geq 2$ points. Detailed variables and evaluation methods are described in table 2.

The study flowchart is depicted in figure 1 .

\section{Statistical analysis plan}

Descriptive analysis of all variables will be performed by appropriate statistical methods. Categorical variables will be analysed using frequency distribution tables (absolute and relative frequencies). Continuous variables will be analysed using sample statistics (ie, mean, SD, minimum,

Table 2 Variables and evaluation methods

\section{Study outcomes \\ Percentages of MRI/ultrasound scores of representative joints improved/unchanged from baseline at the end of the study. \\ ABR, AJBR and ATJBR}

\section{X-ray outcome}

Joint function

Motion

Quality of life

Percentage of patients remaining at each step of administration at Percentage of patients the end of this study

FVIII consumption

Inhibitors

Treatment compliance

\section{Variables and methods}

MRI: IPSG MRI score Ultrasound: HEAD-US score

Annual bleeding and joint bleeding rates

Pettersson score

HJHS

FISH (>7 years old)

CHO-KLAT score, China V.2.0

ABR, annual bleeding rate; AJBR, annual joint bleeding rate; ATJBR, annual target joint bleeding rate; CHOKLAT, Canadian Hemophilia Outcomes-Kids Life Assessment Tool; FISH, functional independence score in haemophilia; HEAD-US, Hemophilia Early Arthropathy Dection with Ultrasound; HJHS, Haemophilia Joint Health Score; IPSG, International Prophylaxis Study Group. 


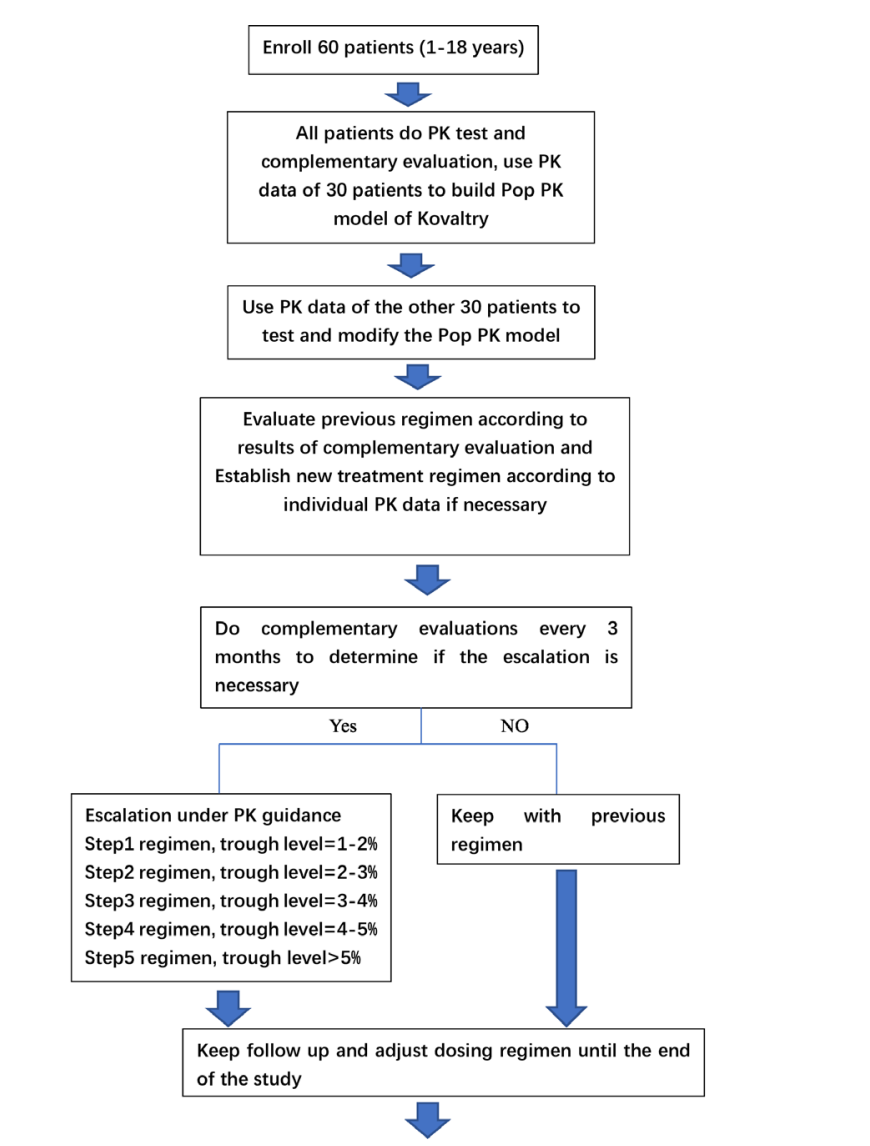

Observe Stage: 2 years

Main endpoints: 1. Changes in MRI/US scores of joints 2. Pop PK model of Kovaltry Secondary endpoints: bleeding rates, joint structure and function, motion, quality of life, FVIII consumption, disease burden, treatment adherence, PK parameters...

Figure 1 The study flowchart. FVIII, factorVIII; PK, pharmacokinetics.

median, quartile and maximum). Continuous variables will be described using absolute values from each time point and represented as changes from baseline (if applicable). The statistical package for social sciences (SPSS) software V.13.0 will be used for statistical processing. Student's t-test will be performed for the analysis of normally distributed data. The $\chi^{2}$ test will be carried out for enumeration data. Non-normally distributed data will be analysed by the rank sum test. $\mathrm{p}<0.05$ will be considered statistically significant.

\section{Ethics and dissemination}

This study has been approved by the Ethics Committee of Beijing Children's Hospital (BCH). Informed consent will be obtained from all boys with severe haemophilia A and their legally authorised guardians. The results will be organised into manuscripts and submitted to peer-review journals as well as international academic meetings. The original data will be stored at Beijing Children's Hospital, and disclosure will only be available on reasonable request by e-mail to the corresponding authors. The main finding will be open to all participants and the Haemophilia Society.

Author affiliations

${ }^{1}$ Hematology Center, Beijing Children's Hospital, Capital Medical University, Beijing, China

${ }^{2}$ Department of Hematology and Oncology, The Children's Hospital, Zhejiang University School of Medicine, National Clinical Research Center for Child Health, Pediatric Leukemia Diagnostic and Therapeutic Technology Research Center of Zhejiang Province, Zhejiang, China

${ }^{3}$ Chengdu Women's and Children's Central Hospital, School of Medicine, University of Electronic Science and Technology of China, Chengdu, China

${ }^{4}$ Department of Hematology, Chengdu Women \& Children's New Century Hospital, Chengdu, China

${ }^{5}$ Department of Hematology, Jiangxi Provincial Children's Hospital, Nanchang, China ${ }^{6}$ Department of Hematology and Oncology, Children's Hospital of Nanjing Medical University School of Medicine, Nanjing, China

${ }^{7}$ Department of Hematology and Oncology, Shenzhen Children's Hospital, Shenzhen, Guangdong, China

${ }^{8}$ Hematologic Disease Laboratory, Hematology Center, Beijing Children's Hospital, Capital Medical University, Beijing, China

Contributors HK proposed the study, defended during ethical review and wrote the manuscript. $\mathrm{RW}$ and $\mathrm{ZC}$ designed the study, applied for funding and reviewed the manuscript. WX, MZ, XL, ZX, YF and CL discussed on the planning, conduct and reporting of this study and reviewed the manuscript.

Funding The current work was in part supported by grants from Research on the application of clinical characteristics of the Beijing Municipal Science and Technology Commission (code Z181100001718182) and Bayer Health Company (grant number 20006429).

Competing interests None declared.

Patient consent for publication Not required.

Provenance and peer review Not commissioned; externally peer reviewed.

Supplemental material This content has been supplied by the author(s). It has not been vetted by BMJ Publishing Group Limited (BMJ) and may not have been peer-reviewed. Any opinions or recommendations discussed are solely those of the author(s) and are not endorsed by BMJ. BMJ disclaims all liability and responsibility arising from any reliance placed on the content. Where the content includes any translated material, BMJ does not warrant the accuracy and reliability of the translations (including but not limited to local regulations, clinical guidelines, terminology, drug names and drug dosages), and is not responsible for any error and/or omissions arising from translation and adaptation or otherwise.

Open access This is an open access article distributed in accordance with the Creative Commons Attribution Non Commercial (CC BY-NC 4.0) license, which permits others to distribute, remix, adapt, build upon this work non-commercially, and license their derivative works on different terms, provided the original work is properly cited, appropriate credit is given, any changes made indicated, and the use is non-commercial. See: http://creativecommons.org/licenses/by-nc/4.0/.

ORCID iD

Huang Kun http://orcid.org/0000-0003-1641-1472

\section{REFERENCES}

1 Hermans C. Guidelines for the prophylaxis of haemophilia A and B: new horizons and ambitions. Br J Haematol 2020;190:643-4.

2 Srivastava A, Santagostino E, Dougall A. Haemophilia. In: WFH guidelines for the management of hemophilia. . 3rd edn, 2020: 6. 1-158.

3 Nilsson IM, Blombäck M, Ahlberg A. Our experience in Sweden with prophylaxis on haemophilia. Bibl Haematol 1968;34:111-24.

4 Nilsson IM, Berntorp E, Löfqvist T, et al. Twenty-Five years' experience of prophylactic treatment in severe haemophilia A and B. J Intern Med 1992;232:25-32.

5 van den Berg HM, Fischer K, Mauser-Bunschoten EP, et al. Longterm outcome of individualized prophylactic treatment of children with severe haemophilia. Br J Haematol 2001;112:561-5.

6 Feldman BM, Pai M, Rivard GE, et al. Tailored prophylaxis in severe hemophilia $A$ : interim results from the first 5 years of the 
Canadian hemophilia primary prophylaxis study. J Thromb Haemost 2006;4:1228-36.

7 Wu R, Luke K-H, Poon M-C, et al. Low dose secondary prophylaxis reduces joint bleeding in severe and moderate haemophilic children: a pilot study in China. Haemophilia 2011;17:70-4.

8 Chen Z-P, Li P-J, Li G, et al. Pharmacokinetic studies of factor VIII in Chinese boys with severe hemophilia A: a single-center study. Chin Med J 2018;131:1780-5.

9 Björkman S, Oh M, Spotts G, et al. Population pharmacokinetics of recombinant factor VIII: the relationships of pharmacokinetics to age and body weight. Blood 2012;119:612-8.

10 Lee CA, Owens D, Bray G. Pharmacokinetics of recombinant factor VIII (recombinate) using one-stage clotting and chromogenic factor VIII assay. Thromb Haemost 1999;82:1644-7.

11 Ragni MV, Croteau SE, Morfini M, et al. Pharmacokinetics and the transition to extended half-life factor concentrates: communication from the SSC of the ISTH. J Thromb Haemost 2018;16:1437-41.

12 Iorio A, Keepanasseril A, Foster G, et al. Development of a WebAccessible population pharmacokinetic Service-Hemophilia (WAPPS-Hemo): study protocol. JMIR Res Protoc 2016;5:e239.
13 Nagao A, Yeung CHT, Germini F, et al. Clinical outcomes in hemophilia A patients undergoing tailoring of prophylaxis based on population-based pharmacokinetic dosing. Thromb Res 2019;173:79-84.

14 Collins PW, Blanchette VS, Fischer K, et al. Break-through bleeding in relation to predicted factor VIII levels in patients receiving prophylactic treatment for severe hemophilia a. J Thromb Haemost 2009;7:413-20.

15 Den UIJL IEM, Mauser Bunschoten EP, Roosendaal G, et al. Clinical severity of haemophilia A: does the classification of the 1950s still stand?. 2011;17:849-53.

16 den Uijl IEM, Fischer K, Van Der Bom JG, et al. Analysis of low frequency bleeding data: the association of joint bleeds according to baseline FVIII activity levels. Haemophilia 2011;17:41-4.

17 Yao W, Li X, Tang L. Results from a prospective, dose escalating, prophylaxis study in young boys with severe hemophilia A in China: the China hemophilia individualized prophylaxis study (chips). Haemophilia 2018.

18 Björkman S. Limited blood sampling for pharmacokinetic dose tailoring of FVIII in the prophylactic treatment of haemophilia a. Haemophilia 2010;16:597-605. 